\title{
TRIORGANOTIN(IV) COMPLEXES DERIVED FROM INTERNALLY FUNCTIONALIZED OXIMES
}

\author{
Vinita Sharma ${ }^{1}$, Rajnish K. Sharma ${ }^{1}$, Rakesh Bohra* ${ }^{1}$ and Vimal K. Jain ${ }^{2}$ \\ ${ }^{1}$ Department of Chemistry, University of Rajasthan, Jaipur-302004, India <rkbohra@satyam.net.in> \\ ${ }_{2}^{2}$ Novel Marrialsand Strucural Chemisty Division, Bhabha Atomic Research Center, Mumbai-400085, India
}

\begin{abstract}
Reactions of triorganotin(IV) chlorides with sodium salt of internally functionallized oximes in $1: 1$ molar ratio in refluxing anhydrous benzene, gives $\mathrm{R}_{3} \mathrm{Sn}[\mathrm{ONC}(\mathrm{Me}) \mathrm{Ar}]$ (where $\mathrm{R}=\mathrm{Me}, \mathrm{Et}, \mathrm{Bu}$ and $\mathrm{Ar}=2$ $\mathrm{NC}_{5} \mathrm{H}_{4}{ }^{-}, \quad 2-\mathrm{OC}_{4} \mathrm{H}_{3-}$ or $2-\mathrm{SC}_{4} \mathrm{H}_{3}-$ ). All these derivatives have been characterized by their elemental analysis, IR and NMR $\left[{ }^{1} \mathrm{H},{ }^{13} \mathrm{C}\left\{{ }^{1} \mathrm{H}\right\}\right.$ and $\left.{ }^{119} \mathrm{Sn}\right]$ spectral studies. Molecular weight measurements show their monomeric behaviour in freezing benzene. On the basis of tin-hydrogen $\left[{ }^{2} \mathrm{~J}\left({ }^{119} \mathrm{Sn},{ }^{1} \mathrm{H}\right)\right]$ and tin-carbon $\left[{ }^{1} \mathrm{~J}\left({ }^{119} \mathrm{Sn},{ }^{13} \mathrm{C}\right)\right]$ coupling constants as well as ${ }^{119} \mathrm{Sn}$ NMR data, a tetrahedral geometry around the tin atom has been proposed through oxygen atom of the ligand moiety.
\end{abstract}

\section{INTRODUCTION}

Recently we have reported the synthesis of some diorganotin(IV) complexes containing internally functionallized oxime moieties and have shown that subtle change in the $\mathrm{R}$ group on tin results in different structural motifs as revealed by the X-ray diffraction studies of $\left[\left\{\mathrm{Et}_{2} \mathrm{Sn}(\mathrm{ONC}(\mathrm{Me}) \mathrm{Py})\right\}_{2} \mathrm{O}\right]_{2}$ (I) and $\left[\left\{\mathrm{Bu}_{2}{ }_{2} \mathrm{Sn}(\mathrm{ONC}(\mathrm{Me}) \mathrm{Py})\right\}_{2} \mathrm{O}\right]_{2}$ (II) $)^{2}$. In the case of (I), the oxime nitrogen is coordinated to the endocyclic tin atom whereas in the case of (II) it is coordinated to the exocyclic tin atom.

Triorganotin(IV) complexes of the type $\mathrm{R}_{3} \mathrm{SnL}$ (where $\mathrm{L}=$ anionic bidentate ligand) have received considerable attention due to their varied coordination chemistry and diversity in their structural patterns. ${ }^{2-10}$ Biocidal activities of several triorganotin(IV) compounds are now well documented and the toxicity is species specific. ${ }^{11-13}$ In addition, some tri-and diorganotin(IV) compounds exhibit anti-tumor activities. ${ }^{14-1 \%}$

Oximes are an important class of multidentate organic ligands capable of forming complexes with a remarkable diversity in coordination pattern. ${ }^{1,20-22}$ They also show a variety of biological applications. ${ }^{23}, 24$

In view of the above and pursuance of our interest on organotins we have synthesized triorganotin(IV) complexes derived from internally functionallized oximes and the results are reported herein.

\section{MATERIALS AND METHODS}

All chemicals were of reagent grade and dried before use. Me

${ }_{3} \mathrm{SnCl}$ and $\mathrm{Bu}{ }_{3} \mathrm{SnCl}$ were used as supplied while $\mathrm{Et}_{3} \mathrm{SnCl}$ was prepared according to the literature method. ${ }^{25}$ Tin was estimated as tin-oxide and nitrogen was estimated as reported in the literature. ${ }^{26}$ For compounds of the type $\mathrm{R}_{3} \mathrm{Sn}\{\mathrm{ONC}(\mathrm{Me}) \mathrm{Py}\}$, the nitrogen present in the aromatic ring does not decompose by this procedure. This fact is further corroborated by carrying out blank estimation of nitrogen in pyridine itself.

Molecular weight measurements were carried out by determining depression in freezing point of benzene, using Beckman's Thermometer (Einsteiithermometer n-Beckmann, Labortherm-N, Skalewert, 0.01 $\mathrm{K}$, made in GDR) fitted in a glass assembly (Supplied by JSGW, India). Calculation was done according to the formula $M=1000 \times K_{f} \times w / W \times \Delta T_{f}$, where $M=$ molecular weight, $w=$ weight of solute in $g m s, K_{f}=$ molar Depression Constant, $\mathrm{W}=$ weight of solvent in $\mathrm{gms}$ and $\Delta \mathrm{T}_{\mathrm{f}}=$ depression of freezing point. IR spectra were recorded as neat liquid on a Bomen MB - $102 \mathrm{FT}-\mathrm{IR}$ spectrophotometer. ${ }^{1} \mathrm{H},{ }^{13} \mathrm{C}\left\{{ }^{1} \mathrm{H}\right\}$ and ${ }^{119} \mathrm{Sn}\left\{{ }^{1} \mathrm{H}\right\}$ NMR spectra were recorded as $\mathrm{CDCl}_{3}$ solutions on a Bruker DPX-300 NMR specrometer operating at 300 , 75.5, $111.9 \mathrm{MHz}$, respectively. Chemical shifts are relative to internal chloroform peak for ${ }^{2} \mathrm{H}$ and ${ }^{13} \mathrm{C}$ and external $\mathrm{Me}_{4} \mathrm{Sn}$ in $\mathrm{C}_{6} \mathrm{D}_{6}$.

Preparation of $\left[\mathrm{Bu}_{3} \mathrm{Sn}\{\mathrm{ONC}(\mathrm{Me}) \mathrm{Py}\}\right]$

To a methanolic solution of sodium salt of the ligand [prepared by the reaction of sodium $(150 \mathrm{mg}$, $6.52 \mathrm{~m} \mathrm{~mol})$ with 2-acetylpyridyl oxime $(892 \mathrm{mg}, 6.55 \mathrm{~m} \mathrm{~mol})$ in refluxing methanol], a benzene solution of $\mathrm{Bu}_{3} \mathrm{SnCl}(2.13 \mathrm{~g}, 6.54 \mathrm{~m} \mathrm{~mol})$ was added and refluxed for $5 \mathrm{~h}$. NaCl formed $(378 \mathrm{mg}, 6.46 \mathrm{~m} \mathrm{~mol})$ during the reaction was filtered off and the filtrate was concentrated in vacuo to give a pinkish liquid which was distilled under vacuo $\left(190^{\circ} \mathrm{C}\right.$, at $0.1 \mathrm{~mm}, 2.50 \mathrm{~g}$, yield $\left.90 \%\right)$ as pink liquid.

All other complexes were prepared by the similar rout, their physical and NMR data are given in tables 1 and 2 , respectively.

\section{RESULTS AND DISCUSSION}

Triorganotin(IV) complexes containing internally functionallized oximes were prepared by the reactions of the sodium salt of the ligands with trialkyltin chloride in refluxing anhydrous benzene: 


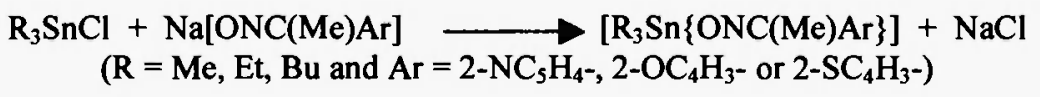

TABLE-1 Synthetic and Analytical data of triorganotin(IV)oximates

\begin{tabular}{|c|c|c|c|c|c|c|}
\hline \multirow[t]{2}{*}{ Complex } & \multirow[t]{2}{*}{ Colour } & \multirow{2}{*}{$\begin{array}{l}\% \text { yield } \\
\text { (After } \\
\text { distillati } \\
\text { on) }\end{array}$} & \multirow{2}{*}{$\begin{array}{l}\text { B. P. } \\
\left({ }^{\circ} \mathrm{Cl} .1\right. \\
\mathrm{mm})\end{array}$} & \multicolumn{2}{|c|}{$\begin{array}{l}\text { \% Analysis } \\
\text { Found (Calcd.) }\end{array}$} & \multirow{3}{*}{$\begin{array}{l}\text { Molec- } \\
\text { ular } \\
\text { weight } \\
\text { Found } \\
\text { (Calcd.) } \\
297 \\
\text { (298.9) }\end{array}$} \\
\hline & & & & $\mathrm{N}$ & Sn & \\
\hline $\mathrm{Me}_{3} \mathrm{Sn}\{\mathrm{ONC}(\mathrm{Me}) \mathrm{Py}\}(\mathbf{1})$ & Colourless & 96 & $155^{\circ} \mathrm{C}$ & $\begin{array}{l}4.5 \\
(4.7)^{\mathrm{a}}\end{array}$ & $\begin{array}{l}39.5 \\
(39.7)\end{array}$ & \\
\hline $\mathrm{Et}_{3} \mathrm{Sn}\{\mathrm{ONC}(\mathrm{Me}) \mathrm{Py}\}(2)$ & Pink & 90 & $160^{\circ} \mathrm{C}$ & $\begin{array}{l}3.9 \\
(4.1)^{\mathrm{a}}\end{array}$ & $\begin{array}{l}34.6 \\
(34.8)\end{array}$ & $\begin{array}{l}342 \\
(340.9)\end{array}$ \\
\hline $\mathrm{Bu}_{3}{ }_{3} \mathrm{Sn}\{\mathrm{ONC}(\mathrm{Me}) \mathrm{Py}\}(3)$ & Pink & 90 & $190^{\circ} \mathrm{C}$ & $\begin{array}{l}3.4 \\
(3.3)^{\mathrm{a}}\end{array}$ & $\begin{array}{l}27.8 \\
(27.9)\end{array}$ & $\begin{array}{l}412 \\
(425.0)\end{array}$ \\
\hline $\begin{array}{l}\mathrm{Me}_{3} \mathrm{Sn}\left\{\mathrm{ONC}(\mathrm{Me}) \mathrm{C}_{4} \mathrm{H}_{3} \mathrm{O}\right\} \\
\text { (4) }\end{array}$ & Colourless & 96 & $115^{\circ} \mathrm{C}$ & $\begin{array}{l}4.7 \\
(4.8)\end{array}$ & $\begin{array}{l}41.1 \\
(41.2)\end{array}$ & $\begin{array}{l}281 \\
(287.8)\end{array}$ \\
\hline $\begin{array}{l}\mathrm{Et}_{3} \mathrm{Sn}\left\{\mathrm{ONC}(\mathrm{Me}) \mathrm{C}_{4} \mathrm{H}_{3} \mathrm{O}\right\} \\
\text { (5) }\end{array}$ & yellow & 96 & $130^{\circ} \mathrm{C}$ & $\begin{array}{l}4.1 \\
(4.2)\end{array}$ & $\begin{array}{l}35.8 \\
(36.0)\end{array}$ & $\begin{array}{l}312 \\
(329.9)\end{array}$ \\
\hline $\begin{array}{l}\mathrm{Bu}_{3}{ }_{3} \mathrm{Sn}\left\{\mathrm{ONC}(\mathrm{Me}) \mathrm{C}_{4} \mathrm{H}_{3} \mathrm{O}\right\} \\
(6)\end{array}$ & yellow & 87 & $210^{\circ} \mathrm{C}$ & $\begin{array}{l}3.3 \\
(3.4)\end{array}$ & $\begin{array}{l}28.5 \\
(28.6) \\
\end{array}$ & $\begin{array}{l}435 \\
(413.9) \\
\end{array}$ \\
\hline $\begin{array}{l}\mathrm{Me}_{3} \mathrm{Sn}\left\{\mathrm{ONC}(\mathrm{Me}) \mathrm{C}_{4} \mathrm{H}_{3} \mathrm{~S}\right\} \\
\text { (7) }\end{array}$ & Colourless & 96 & $135^{\circ} \mathrm{C}$ & $\begin{array}{l}4.5 \\
(4.6)\end{array}$ & $\begin{array}{l}38.8 \\
(39.0)\end{array}$ & $\begin{array}{l}291 \\
(303.8)\end{array}$ \\
\hline
\end{tabular}

"The ring nitrogen of the oxime could not be decomposed by the Kjeldahl method.Therefore, the calculated $\mathrm{N} \%$ relates only to the oxime nitrogen.

Triorganotin(IV) oximates, $\left[\mathrm{R}_{3} \operatorname{Sn}\{\mathrm{ONC}(\mathrm{Me}) \mathrm{Ar}\}\right]$, are faint coloured liquids which can be distilled in vacuo. The boiling point of all these complexes increases with increasing the chain length of the alkyl group on tin atom. Molecular weight measurements in freezing benzene, demonstrate these complexes to be monomeric.

IR spectra of these compounds were interpreted by comparing with the spectra of free oximes as well as with other triorgaotin(IV) oximates. ${ }^{1,2}$ Hydroxy group absorption (appears in the region $3100-3300 \mathrm{~cm}^{-1}$ in the free oximes) is absent in the IR spectra of all these complexes indicating metal-ligand bond formation through oxygen atom. A medium intensity absorption of $\mathrm{C}=\mathrm{N}$ bond in the region $1557-1666 \mathrm{~cm}^{-1}$ and strong $\mathrm{N}-\mathrm{O}$ absorption in the region $900-936 \mathrm{~cm}^{-1}$ for free oximes, are shifted to the lower wave numbers and on complexation are observed in the region $1522-1635 \mathrm{~cm}^{-1}$ and $892-931 \mathrm{~cm}^{-1}$, respectively. The appearance of a new medium to weak intensity band at $467-513 \mathrm{~cm}^{-1}$, may be assigned to $v_{\mathrm{Sn}-0}$ mode. Strong absorption in the region $543-578 \mathrm{~cm}^{-1}$ may be assigned to $v_{\mathrm{Sn}-\mathrm{C}}$. For the trimethyltin(IV) derivatives, the presence of two bands at $\sim 544$ and $513 \mathrm{~cm}^{-1}$ assigned to sym(Sn-Me) and antisym(Sn-Me),suggest a tetrahedral orientation of the Sn-Me bonds.

${ }^{1} \mathrm{H}$ and ${ }^{13} \mathrm{C}\left\{{ }^{1} \mathrm{H}\right\}$ NMR spectra of all these complexes exhibit characteristic signals and multiplicities for R-Sn and ligand protons $\mathrm{s}^{1,21}$ and carbons. In the ${ }^{1} \mathrm{H}$ NMR spectra of all these compounds the hydroxyl proton resonances of free ligand are absent, indicating deprotonation of hydroxyl group of the oxime and concomitant formation of $\mathrm{Sn}-\mathrm{O}$ bond. No appreciable shift has been observed for the ligand ring proton resonances, ruling-out the possibility of coordination through hetero atom present on the aromatic ring of ligand moiety,possibly due to the opposite orientation of the substituents. Similarly, in the ${ }^{13} \mathrm{C}$ NMR spectra, a slight down-field shift $(\delta 0.2-2.4 \mathrm{ppm})$ has been observed for the $\mathrm{C}=\mathrm{N}$ carbon signal. Since, no significant shift in the hetero-aryl ring carbon/proton resonances was observed, it may be inferred that tinhetero atom interactions are absent. The $\mathrm{Me}_{3} \mathrm{Sn}$ complexes displayed a singlet with ${ }^{2} \mathrm{~J}\left({ }^{119} \mathrm{Sn},{ }^{1} \mathrm{H}\right)$ of $56 \mathrm{~Hz}$. Similarly, the ${ }^{13} \mathrm{C}\left\{{ }^{1} \mathrm{H}\right\}$ NMR spectra of these compounds exhibited characteristic peaks for $\mathrm{R}_{3} \mathrm{Sn}$ carbons with ${ }^{1} \mathrm{~J}\left({ }^{119} \mathrm{Sn},{ }^{13} \mathrm{C}\right)$ of $351-393 \mathrm{~Hz}$. The $\mathrm{C}-\mathrm{Sn}-\mathrm{C}$ angle may be calculated from these coupling constants using the following relationship ${ }^{27}$ :

$$
{ }^{1} \mathrm{~J}\left({ }^{119} \mathrm{Sn},{ }^{13} \mathrm{C}\right)=11.4 \theta(\mathrm{C}-\mathrm{Sn}-\mathrm{C})-875
$$

The above relationship give an average value of $109^{\circ}$ for $\mathrm{C}-\mathrm{Sn}-\mathrm{C}$ angles, suggesting tetrahedral geometry around tin atom, which is further substantiated by ${ }^{119} \mathrm{Sn}$ NMR chemical shifts. The ${ }^{119} \mathrm{Sn} \mathrm{NMR}$ spectra of all these compounds displayed a single resonance in the region $\delta 110-150 \mathrm{ppm}$, which is the absorption region for tetra-coordinated triorganotin(IV) atom. 
TABLE-2 ${ }^{1} \mathrm{H},{ }^{13} \mathrm{C}\left\{{ }^{1} \mathrm{H}\right\}$ and ${ }^{119} \mathrm{Sn}\left\{{ }^{1} \mathrm{H}\right\}$ NMR data of triorganotin(IV) oximates in $\mathrm{CDCl}_{3}$

\begin{tabular}{|c|c|c|c|c|}
\hline $\begin{array}{l}\text { Com- } \\
\text { plex } \\
\text { No. }\end{array}$ & 'H NMR $\delta$ & ${ }^{13} \mathrm{C}\left\{{ }^{1} \mathrm{H}\right\}$ NMR $\delta$ & $\begin{array}{c}{ }^{119} \mathrm{Sn} \\
\left\{{ }^{1} \mathrm{H}\right\} \\
\text { NMR } \delta\end{array}$ & $\begin{array}{c}\text { Estimated } \\
\text { C-Sn-C } \angle \\
\text { in }^{\circ}\end{array}$ \\
\hline (1) & $\begin{array}{l}0.50\left(\mathrm{~s},{ }^{2} \mathrm{~J}\left({ }^{119} \mathrm{Sn},{ }^{1} \mathrm{H}\right)=56 \mathrm{~Hz},\right. \\
\mathrm{Sn}-\mathrm{CH}) ; 2.33(\mathrm{~s}, \text { oxime-Me); } \\
7.14(\mathrm{t}), 5.7 \mathrm{~Hz}, \mathrm{H}-4) ; 7.56(\mathrm{t}, 7.6 \\
\mathrm{Hz}, \mathrm{H}-5) ; 7.85(\mathrm{~d}, 7.8 \mathrm{~Hz}, \mathrm{H}-3) ; \\
8.54(\mathrm{~s}, \mathrm{H}-6) .\end{array}$ & $\begin{array}{l}-3.7\left[{ }^{1} \mathrm{~J}\left({ }^{119}{ }^{19} \mathrm{Sn},{ }^{13} \mathrm{C}\right)=387 \mathrm{~Hz}, \mathrm{Sn}-\right. \\
\left.\mathrm{CH}{ }_{3}\right] ; \quad 9.9 \text { (oxime-Me); } 120.1 \\
(\mathrm{C}-5) ; 122.6(\mathrm{C}-3) ; 135.6(\mathrm{C}-4) ; \\
148.5(\mathrm{C}-6) ; 155.8(\mathrm{C}-2) ; 157.5 \\
(\mathrm{C}=\mathrm{N}) \text {. }\end{array}$ & 149.7 & 110.7 \\
\hline (2) & $\begin{array}{l}1.16-1.44\left(\mathrm{~m}, \mathrm{Sn}-\mathrm{CH}_{2} \mathrm{CH}_{3}\right) \\
2.34(\mathrm{~s}, \text { oxime-Me); } 7.17 \text { (br, H- } \\
4) ; 7.60(\mathrm{br}, \mathrm{H}-5) ; 7.88 \text { (d, } 7.8 \\
\mathrm{~Hz}, \mathrm{H}-3) ; 8.56(\mathrm{~s}, \mathrm{H}-6)\end{array}$ & $\begin{array}{l}7.0\left[{ }^{1} \mathrm{~J}\left({ }^{119} \mathrm{Sn},{ }^{13} \mathrm{C}\right)=365 \mathrm{~Hz}, \mathrm{Sn}-\right. \\
\left.\mathrm{CH}_{2}\right] ; 9.7(\mathrm{Sn}-\mathrm{CCH}) ; 9.8 \\
\text { (oxime-Me); } 120.0(\mathrm{C}-5) ; 122.5 \\
(\mathrm{C}-3) ; 135.6(\mathrm{C}-4) ; 148.6(\mathrm{C}-6) ; \\
156.4(\mathrm{C}-2) ; 157.8(\mathrm{C}=\mathrm{N})\end{array}$ & 111.7 & 108.7 \\
\hline (3) & 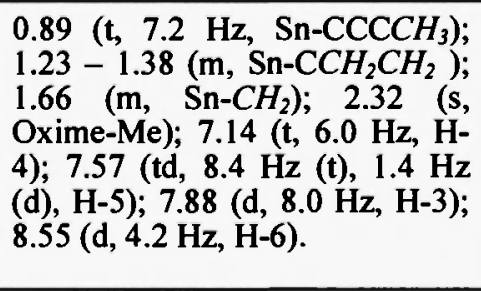 & 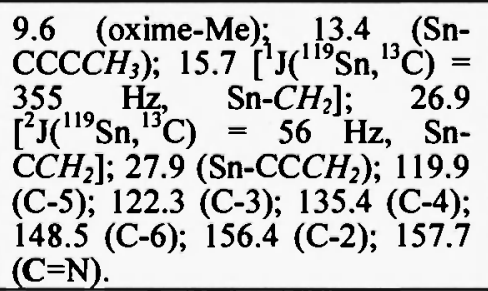 & 115.9 & 107.9 \\
\hline (4) & $\begin{array}{l}0.52\left[\mathrm{~s},{ }^{2} \mathrm{~J}\left({ }^{119} \mathrm{Sn},{ }^{1} \mathrm{H}\right)=56 \mathrm{~Hz}\right. \\
\left.\mathrm{Sn}-\mathrm{CH}_{3}\right] ; 2.17 \text { (oxime-Me); } 6.40 \\
(\mathrm{~s}, \mathrm{H}-4) ; 6.54(\mathrm{br}, \mathrm{H}-3) ; 7.42(\mathrm{~s}, \\
\mathrm{H}-5) .\end{array}$ & $\begin{array}{l}-3.7\left[{ }^{1} \mathrm{~J}\left({ }^{119} \mathrm{Sn},{ }^{13} \mathrm{C}\right)=393 \mathrm{~Hz}, \mathrm{Sn}-\right. \\
\left.\mathrm{CH}_{3}\right] ; 10.7 \text { (oxime-Me); } 107.2 \\
(\mathrm{C}-4) ; 111.0(\mathrm{C}-3) ; 142.6(\mathrm{C}-5) ; \\
149.1(\mathrm{C}-2) ; 152.0(\mathrm{C}=\mathrm{N})\end{array}$ & 149.5 & 111.2 \\
\hline (5) & $\begin{array}{l}1.09-1.46\left(\mathrm{~m}, \mathrm{Sn}-\mathrm{CH}_{2} \mathrm{CH}_{3}\right) \\
2.17 \text { (s, oxime-Me); } 6.38(\mathrm{~s}, \mathrm{H}- \\
4) ; 6.51 \text { (br s, H-3); } 7.39 \text { (s, H- } \\
\text { 5). }\end{array}$ & $\begin{array}{l}6.9\left[{ }^{1} \mathrm{~J}\left({ }^{119} \mathrm{Sn},{ }^{13} \mathrm{C}\right)=354 \mathrm{~Hz}, \mathrm{Sn}-\right. \\
\left.C H_{2}\right] ; 9.6 \quad\left(\mathrm{Sn}-\mathrm{C} C H_{3}\right) ; 10.3 \\
\text { (oxime-Me); } 106.6(\mathrm{C}-4) ; 111.0 \\
(\mathrm{C}-3) ; 142.2(\mathrm{C}-5) ; 149.3(\mathrm{C}-2) ; \\
152.5(\mathrm{C}=\mathrm{N}) .\end{array}$ & 110.9 & 107.8 \\
\hline (6) & $\begin{array}{l}0.91\left(\mathrm{t}, 7.2 \mathrm{~Hz}, \mathrm{Sn}-\mathrm{CCCCH}_{3}\right) ; \\
\left.1.23-1.42 \mathrm{(m}, \mathrm{Sn}-\mathrm{CCCH} \mathrm{CH}_{2}\right) ; \\
1.66 \quad\left(\mathrm{~m}, \mathrm{Sn}-\mathrm{CH}_{2}\right) ; 2.16 \quad(\mathrm{~s}, \\
\text { oxime-Me); } 6.38(\mathrm{br} \quad \mathrm{s}, \mathrm{H}-4) ; \\
6.50(\mathrm{br}, \mathrm{H}-3) ; 7.39(\mathrm{~s}, \mathrm{H}-5)\end{array}$ & 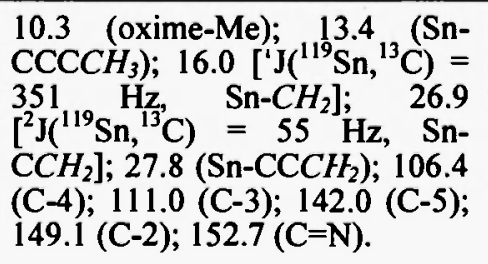 & 114.4 & 107.5 \\
\hline (7) & $\begin{array}{l}0.54\left\{\mathrm{~s},{ }^{2} \mathrm{~J}\left({ }^{119} \mathrm{Sn},{ }^{1} \mathrm{H}\right)=56 \mathrm{~Hz},\right. \\
\left.\mathrm{Sn}-\mathrm{CH}_{3}\right\} ; 2.26(\mathrm{~s}, \text { oxime-Me); } \\
6.99(\mathrm{br}, \mathrm{H}-4) ; 7.15 \text { (br, H-3 and } \\
\mathrm{H}-5) .\end{array}$ & $\begin{array}{l}-3.9,\left[{ }^{1} \mathrm{~J}^{119}{ }^{119} \mathrm{Sn},{ }^{13} \mathrm{C}\right)=393 \mathrm{~Hz}, \\
\left.\mathrm{Sn}-\mathrm{CH} H_{3}\right] ; 11.5 \quad(\mathrm{~s}, \text { oxime-Me); } \\
124.2 \text { (C-4); } 125.3 \quad(\mathrm{C}-3) ; 126.6 \\
(\mathrm{C}-5) ; \quad 142.6 \quad \text { (C-2); } 152.0 \\
(\mathrm{C}=\mathrm{N}) .\end{array}$ & 150.6 & 111.2 \\
\hline
\end{tabular}

Conclusion

On the basis of the above data, the following structure (III) may be proposed for these triorganotin(IV) compounds derived from internally functionalized oximes

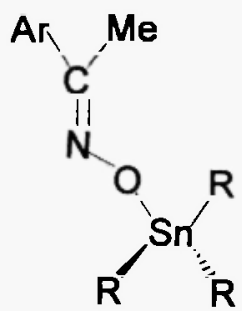

III $\left(\mathrm{R}=\mathrm{Me}, \mathrm{Et}, \mathrm{Bu}\right.$ and $\mathrm{Ar}=2-\mathrm{NC}_{5} \mathrm{H}_{4}-, 2-\mathrm{OC}_{4} \mathrm{H}_{3}-$ or $\left.2-\mathrm{SC}_{4} \mathrm{H}_{3}-\right)$ 


\section{ACKNOWLEDGEMENT}

Two of the authors (V.S. and R.K.S.) are thankful to DAE, Mumbai for the award of fellowships.

Financial support from DAE (Mumbai), UGC and DST (Delhi) is gratefuly acknowledged.

\section{REFERENCES}

1. V. Sharma, R.K. Sharma, R. Bohra, R. Ratnani, V.K. Jain, J.E. Drake, M.B. Hursthouse, M.E. Light, J. Organomet. Chem. (in press) (2002).

2. H. Karel, J. Holecek, M. Nadvornik, A. Lycka, Sb. Ved. Pr.-Vys. Sk. Chemikotechnol. Pardubice, 56, 59 (1992), CA; 121, 134290v, (1994).

3. B.C. Das, G. Biswas, B.B. Maji, K.L. Ghatak, S.N. Ganguli, Y. litaka, A. Benerjee, Acta. Crystallogr., C49, $216(1993)$.

4. V.K. Jain, Coord. Chem. Rev., 135/136, 809 (1994).

5. (a) K. K. Sharma, T.S.B. Baul, E. Rivarola, R.P. Agarwal, Polyhedron, 13, 2217 (1994). (b) A. Badshah, M. Danish, S. Ali, M. Mazhar, S. Mahmood, M.I. Chaudhary, Synth. React. Inorg. Met.-Org. Chem., 24, 1155 (1994). (c) B.D. Liu, B. Ming, J.P. Zhang, Gaodeng Xuexiao Huaxue Xucbao, 15, 1178 (1994), CA, 123, 9555a (1995).

6. (a) S. W. Ng, V.G.K. Das, Main Group Met. Chem., 18, 309 (1995). (b) L.E. Khoa, N. K. Goh, L.L. Koh, Y. Xu, S.L. Bao, T.C.W. Mak, Polyhedron, 14, 2281 (1995).

7. (a) A. Chakrabarti, S. Kamaruddin, T.K. Chattopadhyay, A. Roy, B.N. Chakrabarty, K.C. Molloy, E.R.T. Tiekink, Appl. Organomet. Chem., 9, 357 (1995). (b) M. Danish, H.G. Alt, A. Badshah, S. Ali, M. Mazhar, U.I. Nazar, J. Organomet. Chem., 486, 51 (1995).

8. (a) V.K. Jain, Proc. Indian Acad. Sci (Chem. Sci.), 108, 165 (1996). (b) K.M. Attar, Appl. Organomet. Chem., 10, 317 (1996).

9. M.A. Beswick, J.S. Palmer, D.S. Wright, Chem. Soc. Rev., 27, 225 (1998).

10. M. Kammer, L. Ghys, M. Gielen, M. Biesemans, E.R.T. Tiekink, R. Willem, J. Organomet. Chem., 582, 195 (1999).

11. M. Gielen, A.EI Khloufi, M. Biesemans, A. Bouhdid, D. de Vos, B. Mahieo, R. Willem, Met. Based. Drugs, 1, 305 (1994).

12. Q. Xie, Y. Zhu, G.Liu, Yingyong Huaxue, 12, 79 (1995).

13. A. Jain, S. Saxena, A.K. Rai, P.N. Saxena, J.V. Rao, Metal-Based Drugs, 6, 183 (1999).

14. G. Lhuang, S.G. Dai, H.W. Sun, Appl. Organomet. Chem., 10, 377 (1996).

15. G. Eng, D. Whalen, Y.Z. Zhang, J. Tierney, X.L. Jiang, L. May, Appl. Organomet. Chem., 10, 495 (1996).

16. S.K. Kamruddin, T.K. Chattopadhyaya, A. Roy, E.R.T. Tiekink, Appl. Organomet. Chem., 10, 513 (1996).

17. L. Pellerito, F. Maggio, T. Fiore, A. Pellerito, Appl. Organomet. Chem. 10, 393 (1996).

18. M. Gianguzza, G. Dolcemascolo, C. Mansueto, L. Pelleito, Appl. Organomet. Chem., 10, 405 (1996).

19. M. Gielen, H. Dalil, M. Biesemans, B. Mahieu, D. vos De, R. Willem. Appl. Organomet. Chem., 13, 515 (1999).

20. (a) F. Kayser, M. Biesemans, M. Boualam, E.R.T. Tiekink, A. EI Khloufi, J. Meunier-Piret, A. Bouhdid, K. Jurkschat, M. Gielen, R. Willem, Organometallics, 13, 1098 (1994). (b) A. Meddour, A. Bouhdid, M. Gielen, M. Biesemans, F. Mercier, E.R.T. Tiekink, R. Willem, Eur. J. Inorg. Chem., 1467 (1998).

21. A. Gupta, R.K. Sharma, R. Bohra, V.K. Jain, J.E. Drake, M.B. Hursthouse, M.E. Light, J. Organomet. Chem., 645, 118 (2002).

22. N. Sharma, R.K. Sharma, R. Bohra, Main Group Met. Chem., 24, 781 (2001).

23. (a) H.A. Kists, E.F. Szymanski, D.E. Dorman, J. Antibiotics, 28, 286 (1975). (b) K.V. Domasevich, N.N.Gerasimchuk, Russ. J. Inorg. Chem., 37, 3207 (1992).

24. (a) V.V. Ponomareva, N.K. Dalley, Xiaolan Kou, N.N. Gerasimchuk, K.V. Domasevich, J. Chem. Soc. Dalton Trans., 2351 (1996). (b) R. Willem, A. Boudid, F. Kayser, A. Delmotte, M. Gielen, C.J. Martins, M. Biesemans, B. Mahieu, E.R.T. Tiekink, Organometallics, 15, 1920 (1996).

25. H.H. Anderson, Inorg. Chem., 1, 647 (1962); CA, 57, 9868.

26. A.I. Vogel, Text Book of Quantitative Chemical Analysis, Longmans, London ( $V^{\text {tn }}$ Edition) (1989).

27. T.P. Lockhart, W.F. Manders, J.J. Zuckerman, J. Am. Chem. Soc., 107, 4546 (1985).

Received: March 6, 2002 - Accepted: March 18, 2002 Accepted in publishable format: March 26, 2002 